\title{
Quantum trajectories in atom-surface scattering with single adsorbates: The role of quantum vortices
}

\author{
A. S. Sanz ${ }^{\mathrm{a})}$ and F. Borondo \\ Departamento de Química, C-IX, Universidad Autónoma de Madrid, Cantoblanco-28049 Madrid, Spain \\ S. Miret-Artés ${ }^{\text {b) }}$ \\ Instituto de Matemáticas y Física Fundamental, Consejo Superior de Investigaciones Científicas, \\ Serrano 123, 28006 Madrid, Spain
}

(Received 15 October 2003; accepted 23 January 2004)

\begin{abstract}
In this work, a full quantum study of the scattering of He atoms off single $\mathrm{CO}$ molecules, adsorbed onto the $\mathrm{Pt}(111)$ surface, is presented within the formalism of quantum trajectories provided by Bohmian mechanics. By means of this theory, it is shown that the underlying dynamics is strongly dominated by the existence of a transient vortitial trapping with measurable effects on the whole diffraction pattern. This kind of trapping emphasizes the key role played by quantum vortices in this scattering. Moreover, an analysis of the surface rainbow effect caused by the local corrugation that the $\mathrm{CO}$ molecule induces on the surface, and its manifestation in the corresponding intensity pattern, is also presented and discussed. (C) 2004 American Institute of Physics.
\end{abstract}

[DOI: $10.1063 / 1.1683136]$

\section{INTRODUCTION}

In surface scattering, diffraction experiments are of paramount importance. ${ }^{1-3}$ Low-energy beams of light neutral particles are a nondestructive technique that allows one to probe the outermost layers of metallic, insulator, or semiconductor surfaces. In particular, in experiments with $\mathrm{He}$ atoms at low incident energy, diffraction is predominant and the scattering is mainly elastic. This fact has led to a wealth of experimental data, from which a great deal of information about the orientation and size of the surface unit cells, surface corrugation, particle-surface physisorption potentials, surface diffusion, condensation, or growth phenomena, for example, has been obtained. ${ }^{4}$ On the other hand, inelastic processes can also be analyzed: Phonon dispersion curves are determined by combining pulsed beams and time-of-flight techniques, ${ }^{5,6}$ and an attenuation of the diffraction intensities through the well-known Debye-Waller exponential factor (without changing their shapes) is observed. Furthermore, if heavier probe particles (atoms or molecules) are used as scattering particles, multiphoton events become more relevant apart from adding inelastic channels coming from the internal degrees of freedom (rotation and vibration) of the incoming molecules. Surface trapping, resonances, and subsequent desorption $^{7-9}$ can be also assisted by means of inelastic events.

Opposite to well ordered surfaces, defects disrupting their periodicity can also be found. In this case, when $\mathrm{He}$ atoms probe the surface, the corresponding angular distribution of the intensity displays maxima in between the Bragg peaks. This type of scattering is known as diffuse elastic scattering, or incoherent elastic scattering, since no phase

\footnotetext{
a)Present address: Chemical Physics Theory Group, Department of Chemistry, University of Toronto, Toronto, Canada M5S 3H6.

${ }^{b)}$ Electronic mail: s.miret@imaff.cfmac.csic.es
}

relationship exists among particles scattered from different defects. Oscillations at large diffraction angles in the intensity pattern due to elastic scattering of He atoms off randomly stepped Pt(111) surfaces, and estimations of the size of single CO molecules [adsorbed on $\mathrm{Pt}(111)$ surfaces] were reported a long time ago. ${ }^{10}$ After these earlier studies, the $\mathrm{He}-\mathrm{CO} / \mathrm{Pt}(111)$ scattering has become a prototype system for both experimentalists and theoreticians. Observations are better made at low surface temperatures and coverages to prevent the formation of ordered islands, and reduce the inelastic background contribution. Even more, it is known that $\mathrm{CO}$ molecules adsorb mainly at step sites. On the other hand, while working at high incident energies of the incoming $\mathrm{He}$ atoms is good for theory, it is not so convenient experimentally since the inelastic contribution is increased and the resolution is poorer. The different methods used so far to study, from a theoretical point of view, the scattering of atoms off single isolated adsorbates are mainly based on hardwall models, ${ }^{10-12}$ sudden approximation schemes, ${ }^{13}$ quantum wave packets propagation, ${ }^{11,14}$ standard close-coupling gas phase approaches, ${ }^{15}$ and atom-surface scattering formalisms. ${ }^{16}$

Angular distributions are usually measured as a function of the parallel momentum transfer, $\Delta K$, for a given initial impact direction of $\mathrm{He}$ atoms. Large values of $\Delta K$ (or, equivalently, large diffraction angles) are mainly influenced by the repulsive part of the interaction potential. Analogously, scattering intensities at small values of $\Delta K$ are dominated by the attractive, long-range part of the potential. Interference oscillations and rainbow peaks are the basic features observed in this type of angular distributions. However, some controversy regarding the assignment of the dynamical origin of the different types of such features has been unavoidable in literature. Small and intermediate momentum transfer undullations observed were initially ex- 
plained by the introduction of the so-called reflection symmetry interference (RSI) in a hard-wall model. ${ }^{10,12}$ Within this theoretical model, the surface is represented as a mirror, and the admolecule as a hemispherical boss on the mirror. Thus, an incident ray can be directly reflected by the hemispherical boss, or doubly reflected first by the boss and then by the mirror. Hence, the scattering amplitude can be expressed as the sum of two contributions: A direct backscattering term (involving mainly the interaction with the adsorbate), and a term arising from a double scattering event. Each one of these two contributions can be again unfolded in two parts: One accounting for small values of $\Delta K$ (Fraunhofer diffraction), and the second one coming from large values of $\Delta K$. Theoretical studies carried out by Yinnon et al. ${ }^{13}$ with a soft potential showed that not all of the oscillations observed could be attributed to Fraunhofer peaks. Rainbow effects, due to the local corrugation induced by the defect, were predicted from their calculations based on classical trajectories. In an ulterior work, Lemoine ${ }^{15}$ corroborated the presence of rainbow peaks by numerically solving the time-dependent Schrödinger equation for the same system. However, in spite of this exhaustive work, arguments based only on classical trajectories ${ }^{13}$ are not conclusive by their own nature and, on the other hand, those based on pure quantum calculations lack intuitive insight.

Bohmian mechanics ${ }^{17,18}$ is a theoretical formalism combining both the predictive accuracy of quantum mechanics and the capability to provide a causal intuitive picture of quantum processes (analogous to classical descriptions, where the individual nature of material particles is fully described). This alternative formalism of quantum mechanics is similar to the hydrodynamical formulation of quantum mechanics (quantum hydrodynamics) proposed by Madelung, ${ }^{19}$ and goes beyond the de Broglie's concept of pilot wave. ${ }^{20}$ Bohmian mechanics provides a different and clear insight into quantum interference processes without any need to abandon the notion of a well-defined trajectory in space and time. Particles are guided by a surrounding wave solution of the time-dependent Schrödinger equation, so that the trajectories of an ensemble of particles reproduce the statistical predictions of the standard quantum mechanics. In the particular case of the $\mathrm{He}-\mathrm{CO} / \mathrm{Pt}(111)$ system, very regular dynamics a priori can be expected for trajectories colliding with the clean Pt surface corresponding, from a hydrodynamical point of view, to a laminar regime. However, the presence of a single adsorbate should induce around its location more turbulent dynamics. Intensity patterns are shown to be strongly affected by the appearance of vortices and the existence of a transient vortitial trapping. Moreover, it is not necessary to introduce any additional semiclassical notion (as the abovementioned RSI) to fully understand the underlying dynamics. The nonlocal character and context dependence of Bohmian mechanics are enough to better interpret the diffraction pattern.

Quantum hydrodynamics is formally related to some important phenomena in quantum mechanics as, for example, the Aharonov-Bohm effect, ${ }^{21}$ superconductivity, ${ }^{22}$ or BoseEinstein condensation. ${ }^{23}$ The quantization conditions leading to the presence of vortices characterizing these phe- nomena follow a similar mathematical treatment. The theory of quantum vortices was described by Dirac in a classical paper, ${ }^{24}$ in which the existence of magnetic monopoles was suggested. A more explicit development of the quantum theory of magnetic monopoles, which constitutes a generalization of the concept of quantum vortex, can be found in Ref. 25. Moreover, the theory of quantum vortices has been widely described in Refs. 26 and 27, for example, in connection with quantum hydrodynamics, and an interesting related application can be found in Ref. 28.

Bohmian mechanics has been recently applied to different scattering problems. Lopreore and $\mathrm{Wyatt}^{29}$ have analyzed the tunneling of wave packets through barriers, elucidating the corresponding mechanism in terms of quantum trajectories; a portion of them, with initial locations in the foremost part of the initial wave packet, overcomes the barrier because they acquire some additional kinetic energy during a boost phase, shortly after the launching. In the atom-surface scattering context, Sanz et al. have applied Bohmian mechanics to reinterpret diffraction patterns ${ }^{30}$ and the rainbow effect. ${ }^{31}$ On the other hand, using a similar analysis based on mixed quantum-classical simulations, Prezhdo and Brooksby ${ }^{32}$ have solved the quantum backreaction problem for the $\mathrm{O}_{2}-\mathrm{Pt}$ reaction, and Gindensperger et al. have reported a study including continuum states, ${ }^{33}$ and a description of rotational diffractive scattering. ${ }^{34}$

According to the purpose of this work, the organization of the article is as follows: In the next section, a brief description of the fundamentals of Bohmian mechanics is given, emphasizing the dynamical origin of quantum vortices. In Sec. III, the main results obtained from the application of Bohmian theory to the $\mathrm{He}-\mathrm{CO} / \mathrm{Pt}(111)$ scattering are presented and discussed. For the sake of clarity, this section has been subdivided into three subsections. In the first one, a brief discussion on the procedure followed to compute the time propagation of both the wave function and the quantum trajectories is presented. In the second, a comparison between the classical and Bohmian dynamics associated with the $\mathrm{He}-\mathrm{CO} / \mathrm{Pt}(111)$ system is shown. Finally, in the third subsection, the analysis of this scattering in terms of ensembles of quantum trajectories is presented, also analyzing the case of a purely repulsive adsorbate directly connected with the absence of rainbow features. Finally, in Sec. IV, the main conclusions extracted from this work are summarized.

\section{FUNDAMENTALS OF BOHMIAN MECHANICS}

The key element of Bohmian mechanics is the introduction of the concept of particle into the quantal description of nature. ${ }^{17}$ As in classical mechanics, the evolution of the system can be understood as the motion of particles following well-defined trajectories. Thus, a quantum system must be seen as a whole constituted by the particle (having a precisely defined position at each time) and the wave function (extended in space and time, and guiding the motion of the particle). The wave function evolution is governed by the time-dependent Schrödinger equation, and to obtain the equations of motion for the particle, one can proceed by writing the wave function in its polar form as 


$$
\Psi(\mathbf{r}, t)=R(\mathbf{r}, t) \mathrm{e}^{\mathrm{i} S(\mathbf{r}, t) / \hbar},
$$

where the real functions $R(\mathbf{r}, t)$ and $S(\mathbf{r}, t)$ account for the amplitude and phase of the wave function, respectively. Then, introducing Eq. (1) into the time-dependent Schrödinger equation,

$$
\mathrm{i} \hbar \frac{\partial \Psi}{\partial t}=\left[-\frac{\hbar^{2}}{2 m} \nabla^{2}+V\right] \Psi,
$$

where $V(\mathbf{r})$ is the external or classical potential, two real coupled equations,

$$
\begin{aligned}
& \frac{\partial R^{2}}{\partial t}+\boldsymbol{\nabla} \cdot\left(R^{2} \frac{\boldsymbol{\nabla} S}{m}\right)=0, \\
& \frac{\partial S}{\partial t}+\frac{(\boldsymbol{\nabla} S)^{2}}{2 m}+V-\frac{\hbar^{2}}{2 m} \frac{\boldsymbol{\nabla}^{2} R}{R}=0,
\end{aligned}
$$

are obtained. Equations (3) and (4) are the conservation of the probability density, and the quantum Hamilton-Jacobi equation, respectively. The latter is similar to its classical counterpart except for the extra term

$$
Q=-\frac{\hbar^{2}}{2 m} \frac{\nabla^{2} R}{R} .
$$

This function is the so-called quantum potential and it can be seen as the carrier of the quantum information transmitted to quantum trajectories. The quantum potential is a measurement of the curvature of the wave function amplitude. The role of $Q$ as a potential, similar to $V$, but from a purely quantum origin, is better understood by rearranging Eq. (4), and defining the velocity field, $\mathbf{v}$, as

$$
\mathbf{v}=\dot{\mathbf{r}}=\frac{\nabla S}{m} .
$$

This expression implies that each particle trajectory is orthogonal to the $S=$ constant manifold. Now, by applying the operator $\boldsymbol{\nabla}$ to Eq. (4), and substituting Eq. (6) in the resulting expression, one obtains

$$
m \frac{\partial \mathbf{v}}{\partial t}+m(\mathbf{v} \cdot \boldsymbol{\nabla}) \mathbf{v}=-\boldsymbol{\nabla}(Q+V) .
$$

This equation can be identified with a generalized Newton's second law,

$$
m \frac{d \mathbf{v}}{d t}=-\nabla(Q+V),
$$

when the Lagrangian time derivative operator,

$$
\frac{d}{d t}=\frac{\partial}{\partial t}+\mathbf{v} \cdot \boldsymbol{\nabla}
$$

is used. In Eq. (8), a quantum force, $-\nabla Q$, acting on the particle is apparent, in addition to the classical force given by $-\nabla V$. Thus, effects different from those derived from the classical potential, $V$, could be expected. It is, in this sense, that Bohmian mechanics provides a general theory of waves and particles that goes beyond classical mechanics, and not a simple step backward to classical theories.

The peculiarity of Bohmian motion arises from the nonclassical effects caused by the quantum potential. For ex- ample, the action of a classical potential on a particle is usually constrained to a neighborhood of such a particle, i.e., classical interactions are local. However, since the quantum potential keeps information about the whole system (as the wave function does), particles feel a long-range interaction that goes beyond their neighborhood, i.e., two distant particles "know" about the motion of the other due to the strong correlation established between them by the quantum potential. Therefore, this long-range, or nonlocal action leads, in the atom-surface scattering context, to the observation of quantum diffraction effects in the Fraunhofer region, ${ }^{30}$ further away from the classical interaction region. On the other hand, due to the dependence of the quantum potential on the quantum state, Bohmian mechanics is context dependent. This property, related also to nonlocality, means that quantum motion is sensitive to each parameter describing a quantum state.

The fact that quantum potential effects can be important in regions where the classical interaction is negligible leads to a necessary redefinition of the meaning of the asymptotic region. Classically, this is regarded as the region where the interaction is negligible $(V \simeq 0)$. However, as inferred from Eq. (8), this definition is not valid in the quantum case since the action of the quantum potential could be still relevant. ${ }^{30,35,36}$ Angular distributions can be obtained once the wave function has reached the classical asymptotic region because, in the momentum representation, the outgoing diffraction channels are already well-defined (in this region, $\left.\hat{H} \simeq \hat{p}^{2} / 2 m\right)$. On the contrary, in the configuration representation, the wave function is not converged yet, and it needs to reach the Fraunhofer region (i.e., the quantum asymptotic region), where the different diffraction peaks are definitely formed. ${ }^{30,35}$ However, the existence of those diffraction maxima also indicates, according to Eq. (5), that particles could experience intense quantum forces between consecutive maxima. Thus, although the quantum asymptotic condition reads as

$$
m \frac{d \mathbf{v}}{d t} \simeq 0,
$$

it fulfills locally, i.e., quantum particles are locally free.

As said above, one of the most relevant properties of this scattering is the existence of a transient vortitial regime and its strong influence on the underlying dynamics. The condition leading to the formation of quantum vortices arises from the complex character of the wave function. This fact implies the nonsingle valuedness of the wave function phase

$$
S^{\prime}(\mathbf{r}, t)=S(\mathbf{r}, t)+2 \pi n \hbar \quad(n=0, \pm 1, \pm 2, \ldots) .
$$

This multivaluedness takes place only in nodal regions, where $\Psi(\mathbf{r}, t)=0$. In such regions, the smoothness of the wave function disappears, and the value of its phase undergoes discrete jumps. Under these conditions, the quantum density current vanishes and the velocity remains undefined (no particle can cross through a nodal region). However, the velocity field around a nodal region is rotational. This can be easily shown as follows. On one hand, the circulation of the velocity field, $\mathbf{v}$, along a closed path, $\mathcal{C}$, is a quantized magnitude 


$$
\oint_{\mathcal{C}} d \mathbf{l} \cdot \mathbf{v}=\oint_{\mathcal{C}} d \mathbf{l} \cdot \frac{\nabla S}{m}=\frac{1}{m} \oint_{\mathcal{C}} d S=\frac{2 \pi n \hbar}{m} .
$$

On the other hand, by applying Stoke's theorem, Eq. (12) can be rewritten as

$$
\int_{\Sigma} d \mathbf{r} \cdot(\boldsymbol{\nabla} \times \mathbf{v})=\frac{2 \pi n \hbar}{m}
$$

where $\Sigma$ is the region enclosed by $\mathcal{C}$. Therefore, vortices appear when $n \neq 0$, in the nodal regions of the wave function, leading to closed streamlines around the nodes, i.e., to a rotational velocity field.

\section{RESULTS AND DISCUSSION}

\section{A. Model and numerical details of the calculations}

A simple two-dimensional, soft potential model, originally proposed by Yinnon et al. ${ }^{13}$ has been chosen to perform quantum trajectory calculations. It consists of the sum of the interaction between $\mathrm{He}$ and the flat $\mathrm{Pt}(111)$ surface and the interaction between $\mathrm{He}$ and the spherically symmetric $\mathrm{CO}$ adsorbate,

$$
V_{\mathrm{He}-\mathrm{CO} / \mathrm{Pt}(111)}(\mathbf{r})=V_{\mathrm{He}-\mathrm{Pt}(111)}(z)+V_{\mathrm{He}-\mathrm{CO}}\left(\mathbf{r}-\mathbf{r}_{\mathrm{CO}}\right) \text {, }
$$

with $\mathbf{r}_{\mathrm{CO}}$ and $\mathbf{r}=(x, z)$ being the position vectors for the $\mathrm{CO}$ center of mass and the He atom, respectively; $x$ and $z$ are the coordinates along the parallel and perpendicular directions with respect to the surface, respectively, and measured relative to the $\mathrm{CO}$ center of mass. The $\mathrm{He}-\mathrm{Pt}(111)$ interaction is represented by a Morse function with $D=4.0 \mathrm{meV}, \alpha$ $=0.6 \mathrm{bohr}^{-1}$, and $z_{m}=2.3 \mathrm{bohr}$. To describe the He-CO interaction, a Lennard-Jones function is chosen with parameters $\epsilon=2.37 \mathrm{meV}$ and $r_{m}=3.5 \AA$. Thus, for this interaction model, the adsorbate is centered at $x=0$, and has an effective radius (at an incident energy of $10 \mathrm{meV}$ ) of $5.4 \mathrm{bohr}$.

In order to simulate with good accuracy the scattering process, an initial incoming plane wave has been used, approximated as a linear superposition of 250 Gaussian wave packets, ${ }^{35}$ according to

$G_{n}(x, z)=\frac{1}{\sqrt{\pi \sigma_{x} \sigma_{z}}} \mathrm{e}^{-\left(x-x_{0}^{n}\right)^{2} / 2 \sigma_{x}^{2}-\left(z-z_{0}^{n}\right)^{2} / 2 \sigma_{z}^{2}+i k_{i}\left(x-x_{0}^{n}\right)}$,

where $\left(x_{0}^{n}, z_{0}^{n}\right)$ gives the initial position of the center of the $n$th wave packet. In our calculations, these wave packets are homogeneously distributed along a distance of $100 \mathrm{bohr}$ (i.e., $\left|x_{0}^{n}\right| \leqslant 50$ bohr), with the same distance, $z_{0}^{n}=19.4 \mathrm{bohr}$, from the clean Pt surface. The width parameters used for each wave packet are $\sigma_{x}=1.58 \mathrm{bohr}$ and $\sigma_{z}=5 \mathrm{bohr}$. This corresponds to introduce approximately 4 Gaussian wave packets in a length equal to $\sigma_{x}$ (the distance between two consecutive centers is 0.4 bohr), what implies a very good overlapping among them. The resulting plane wave is then launched perpendicularly to the surface from $\langle z\rangle_{0}=19.4 \mathrm{bohr}$, where the classical potential is negligible. Two different values of the normal wave vector have been used: $k_{i}=2.32$ and 4.63 bohr $^{-1}$. These correspond to initial energies of $E_{i}=10$ and $40 \mathrm{meV}$, and de Broglie wavelengths $\lambda_{\mathrm{dB}}=2.71$ and 1.36
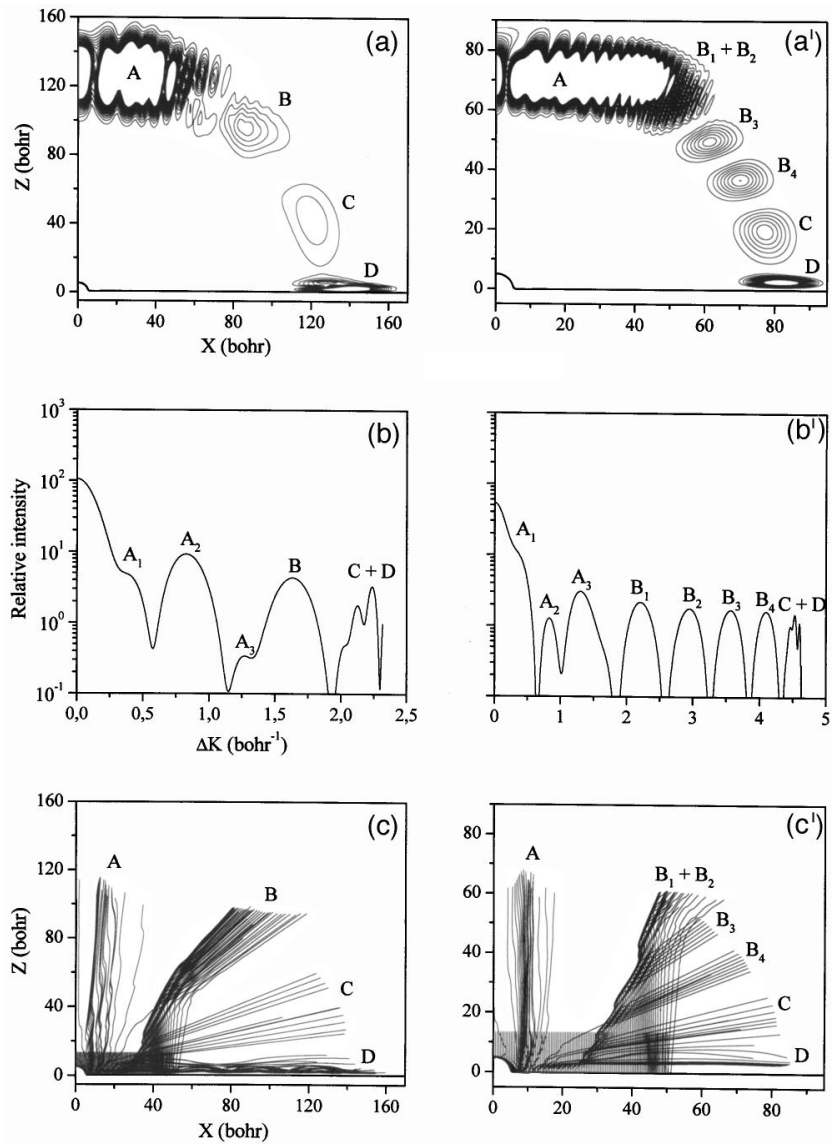

FIG. 1. Comparison of results for $E_{i}=10 \mathrm{meV}$ (left-hand side column) and $E_{i}=40 \mathrm{meV}$ (right-hand side column). Top: probability density at $t=11 \mathrm{ps}$ for $10 \mathrm{meV}$, and $t=3.5 \mathrm{ps}$ for $40 \mathrm{meV}$; the different maxima correlate with peaks in central panels. Center: relative intensity as a function of the parallel momentum transfer, $\Delta K$, at normal incidence. Bottom: quantum trajectories with initial positions (impact parameters) distributed parallel to the clean $\mathrm{Pt}$ surface, and starting at $z_{0}=\langle z\rangle_{0}-6$ bohr.

bohr, respectively. The evolution of the wave function has been computed by standard algorithms, widely described in literature. ${ }^{37-39}$

Bohmian trajectories are obtained by simultaneous numerical integration of Eqs. (2) and (6). The impact parameter associated to each quantum trajectory is assigned according to the following procedure: The region covered by the incoming plane wave can be imaginary divided into parallel "slices" along the $x$ direction (perpendicular to the direction of the wave function propagation), characterized by $z_{0}$ $=\langle z\rangle_{0} \pm \delta$ (the $\pm \delta$ quantity gives the distance from the central slice, $z_{0}=\langle z\rangle_{0}$, of the wave packet, $(+)$ for above or $(-)$ for below); then, the quantum trajectories with initial conditions, $x_{0}$ (i.e., the impact parameters) along each slice are weighted according to the value of the initial probability density at $x_{0}$ and $z_{0}$.

\section{B. Classical mechanics versus Bohmian mechanics}

In Fig. 1, our results for $E_{i}=10 \mathrm{meV}$ (left-hand side column) and $E_{i}=40 \mathrm{meV}$ (right-hand side column) and perpendicular incidence are plotted. In panels (a) and $\left(\mathrm{a}^{\prime}\right)$, the probability densities, $|\Psi(\mathbf{r}, t)|^{2}$, at $11 \mathrm{ps}$ and $3.5 \mathrm{ps}$, respectively, are displayed. The different maxima observed corre- 


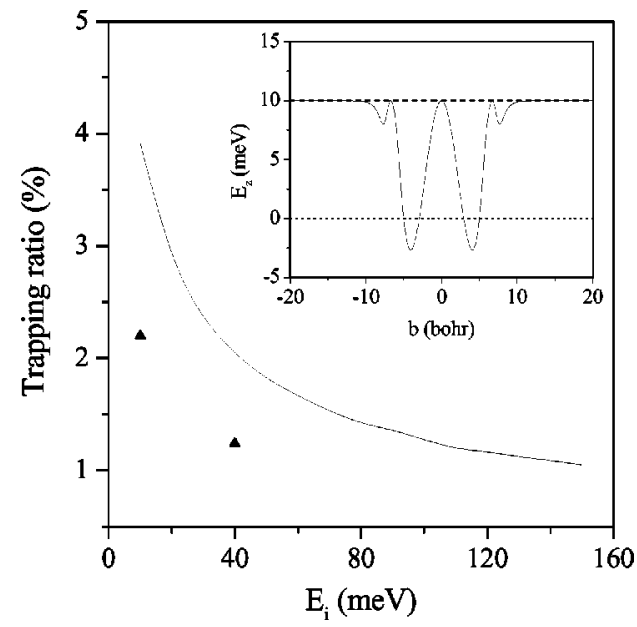

FIG. 2. Classical trapping ratio as a function of the incidence energy $\left(E_{i}\right)$. This ratio is a measurement of the relation between the number of classical trajectories trapped along the surface and the total number of classical trajectories considered. Triangles mark the quantum ratios corresponding to $E_{i}=10 \mathrm{meV}$ and $E_{i}=40 \mathrm{meV}$. In the inset, the asymptotic component (see Ref. 41) of the energy along the $z$ direction as a function of the classical impact parameter, $b$, is also plotted for perpendicular incidence at $10 \mathrm{meV}$.

late to peaks in panels $(b)$ and $\left(b^{\prime}\right)$, which show the intensity as a function of the parallel momentum transfer, $\Delta K$, of the scattering particles. In Fig. 1(b), peaks labeled as $A_{1}, A_{2}$, and $B$ are assigned to Fraunhofer peaks, and $A_{3}$ and the small "shoulder" on the left-hand side of peaks labeled as $C+D$ are related to the presence of classical surface rainbows, since these peaks do not appear in calculations carried out with a purely repulsive adsorbate. The peak $A_{1}$ also disappears although it cannot be assigned to a surface rainbow as explained below. On the other hand, the part of the outgoing wave trapped along the surface [maxima $D$ in (a) and $\left(\mathrm{a}^{\prime}\right)$ ] could be responsible for a resonance phenomenon if an additional adsorbate were found along its way on the surface. This is a well-characterized phenomenon known as defect mediated selective adsorption resonance, predicted by Yinnon et al. ${ }^{13}$ and recently observed by Glebov et al. ${ }^{40} \mathrm{In}$ panels (c) and ( $\left.c^{\prime}\right)$ of Fig. 1, two representative ensembles of quantum trajectories with impact parameters along $z_{0}=\langle z\rangle_{0}$ -6 bohr are shown. Notice that these ensembles represent only a partial contribution to each intensity peak shown in (b) and $\left(\mathrm{b}^{\prime}\right)$, since the total intensity is obtained by considering a larger number of trajectories, densely distributed within the region covered by the initial probability density $\left|\Psi_{0}(\mathbf{r})\right|^{2}$. In panels (c) and ( $\left.\mathrm{c}^{\prime}\right)$, it can be clearly seen how quantum trajectories follow paths of maximum probability, leading to the different maxima of $|\Psi(\mathbf{r}, t)|^{2}$ as shown in panels (a) and $\left(a^{\prime}\right)$, respectively.

At the energies considered here, the percentage of trapped quantum trajectories is $2.20 \%$ for $10 \mathrm{meV}$, and $1.24 \%$ for $40 \mathrm{meV}$, with respect to a total number of 3000 trajectories covering the surface area illuminated by $\left|\Psi_{0}(\mathbf{r})\right|^{2}$. The estimation of these trapping ratios has been done by assigning to each quantum trajectory an appropriate weight according to $\left|\Psi_{0}(\mathbf{r})\right|^{2}$. For comparison, in Fig. 2, the classical trapping ratio as a function of the incident energy, $E_{i}$, has been also plotted showing an exponential-like decay.
The classical ratios corresponding to the incident energies considered in the quantum calculations, $3.91 \%$ and $2.03 \%$, respectively, give a reasonable agreement with their quantum counterparts (triangles). Due to the local character of classical mechanics (unlike Bohmian mechanics), to compute the trapping ratio, it is not necessary to sample the twodimensional region covered by $\left|\Psi_{0}(\mathbf{r})\right|^{2}$ with (classical) trajectories; any two trajectories with the same initial $x_{0}$ coordinate, but different $z_{0}$ (within the classical asymptotic region), will follow the same evolution. An ensemble of 10000 trajectories has been considered to obtain the results shown in Fig. 2. All trajectories were initialized at a distance of 19.4 bohr from the clean Pt surface, and symmetrically distributed with respect to the center of the adsorbate, covering a total length of $100 \mathrm{bohr}$ along the $x$ direction (the same covered by $\left.\left|\Psi_{0}(\mathbf{r})\right|^{2}\right)$. Classical trapped trajectories are characterized by negative values of their (asymptotic) perpendicular energy component. ${ }^{41}$ In the inset of Fig. 2, a classical plot of this component $\left(E_{z}\right)$ as a function of the impact parameter $(b)$ is shown for $E_{i}=10 \mathrm{meV}$. Due to the perpendicular incidence considered, the profile displays two negative lobes symmetrically located with respect to the center of the adsorbate. These two lobes define the set of impact parameters for which (classical) surface trapping dynamics are observed. Under these conditions, and after the first impact with the surface, $\mathrm{He}$ atoms oscillate along the $z$ direction (i.e., show a bound motion inside the well of the Morse function), and move freely along the $x$ direction with an energy greater than $E_{i}\left(E_{x}=E_{i}-E_{z}>E_{i}\right.$, since $\left.E_{z}<0\right)$. The regions of impact parameters leading to trapping decrease when $E_{i}$ increases, indicating the close relation between their sizes and the trapping ratio; a higher impact energy, a lesser number of atoms will remain trapped since they carry enough energy to overcome the attractive well of the Morse function. The two negative lobes mentioned above become deeper and sharper since the increasing in $E_{i}$ implies a larger exchange of momentum. Thus, atoms remain oscillating with higher negative values of $E_{z}$, closer to the depth of the well. Nevertheless, independent of $E_{i}$, it is worth commenting that among the trapped trajectories, that corresponding to the impact parameter belonging to the minimum of the lobe is special. Any trajectory reaches an asymptotic constant oscillation amplitude once the influence of the adsorbate in its dynamics is negligible. However, the trajectory with maximum $\left|E_{z}\right|$ energy displays a constant oscillation amplitude since the first "bounce" of the atom on the surface. Such a trajectory acts as a caustics for double scattering (thus, of relevance for interaction models based on the RSI notion), and can be considered as "stationary" within this context.

The effects of classical surface trapping are also manifested in the quantum dynamics of the system, as can be seen in Figs. 3(a) and 3(b), where the time dependence of the expectation values of $x$ and $z\left(\langle x\rangle_{t}\right.$ and $\langle z\rangle_{t}$, respectively) for the trapped part of the wave function are displayed. The influence of the adsorbate on the wave function is negligible approximately $5 \mathrm{ps}$ after the initial instant. After this time, $\langle x\rangle_{t}$ follows a linear function, trace of a uniform motion of this portion of the wave function along the direction parallel 

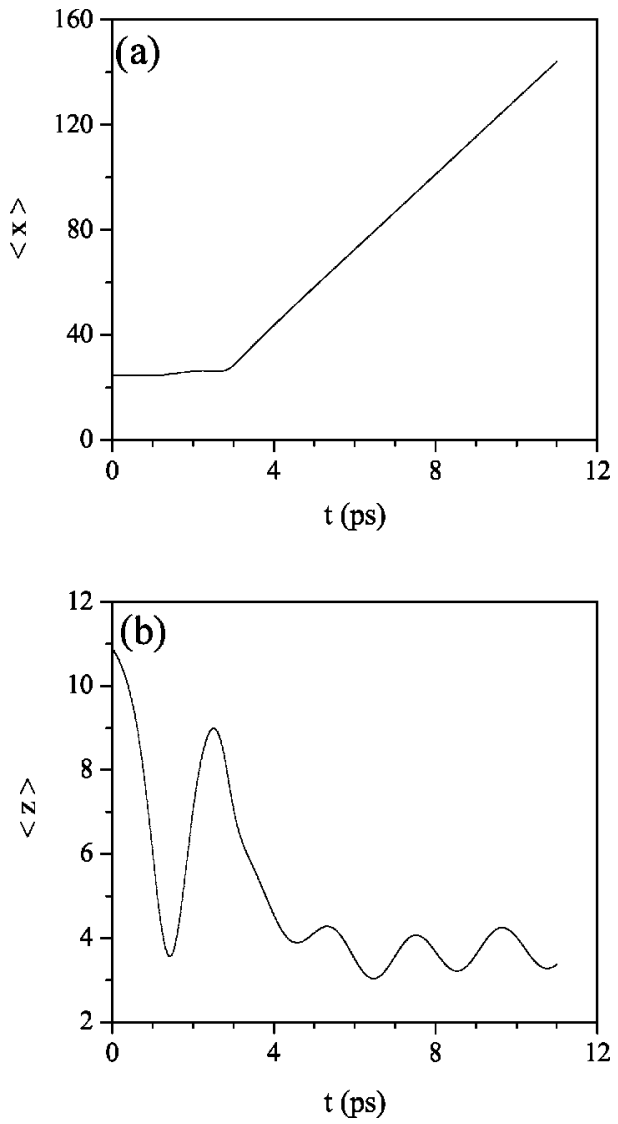

FIG. 3. Expectation value of the position for the trapped ( $D$ portion) wave function as a function of time: (a) $\langle x\rangle$ and (b) $\langle z\rangle$. These expectation values have been computed within the region defined by $x \geqslant 0$ bohr and $z$ $\leqslant 12$ bohr (where classical and quantum motions are bound), and for perpendicular incidence at $10 \mathrm{meV}$.

to the surface $[D$ in Fig. 1(a) $]$. From the slope of this function, the average velocity for $D$ results $\left\langle v_{x}\right\rangle$ $=14.31 \mathrm{bohr} / \mathrm{ps}$, which can be interpreted as the group velocity of the ensemble of trapped Bohmian trajectories. Moreover, the value of the estimated average energy along the $x$ direction is $\left\langle E_{x}\right\rangle=11.89 \mathrm{meV}$. The oscillatory motion of the $D$ portion can also be observed in Fig. 3(b) by means of $\langle z\rangle_{t}$. From this plot, both the frequency of the oscillation and the vibrational energy associated to that portion can be easily calculated. A simple estimation gives $\omega$ $=2.538 \mathrm{ps}^{-1}$, and $\left\langle E_{z}\right\rangle=E_{i}-\left\langle E_{x}\right\rangle=-1.89 \mathrm{meV}$. If the same procedure is followed for $E_{i}=40 \mathrm{meV}$, one finds $\left\langle v_{x}\right\rangle=27.18 \mathrm{bohr} / \mathrm{ps}$ and, therefore, $\left\langle E_{x}\right\rangle=42.91 \mathrm{meV}$ and $\left\langle E_{z}\right\rangle=-2.91 \mathrm{meV}$. These results keep a close relation with those extracted from Fig. 2. Classically, minima appears at $E_{z}^{\mathrm{min}}=-2.68 \mathrm{meV}$ for $E_{i}=10 \mathrm{meV}$, and at $E_{z}^{\min }$ $=-3.00 \mathrm{meV}$ for $E_{i}=40 \mathrm{meV}$. Notice the small difference between $\left\langle E_{z}\right\rangle$ and $E_{z}^{\min }$ in the case of $E_{i}=40 \mathrm{meV}$, what means that the portion of the trapped wave function moves as the (classical) stationary trajectory does. Therefore, as mentioned above, an increasing of the impact energy makes the system to behave more classically. Furthermore, if one takes into account the three bound levels of the Morse potential used, $E_{0}=-2.53 \mathrm{meV}, E_{1}=-0.60 \mathrm{meV}$, and $E_{2}$ $=-3.0 \times 10^{-3} \mathrm{meV}$, one observes that the value of $\left\langle E_{z}\right\rangle$ for $E_{i}=10 \mathrm{meV}$ approaches the ground state, $E_{0}$. Hence, this
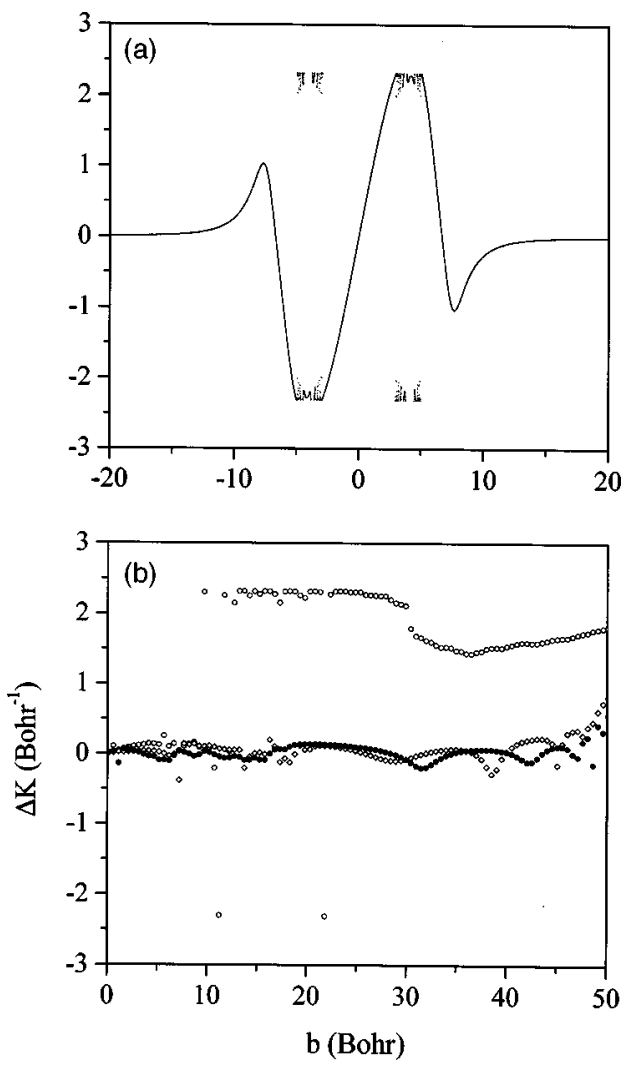

FIG. 4. Classical (a) and quantum (b) deflection functions. The quantum deflection function has been computed for three different ensembles of Bohmian trajectories, beginning at $z_{0}=\langle z\rangle_{0}-6$ bohr (O), $z_{0}=\langle z\rangle_{0}$ bohr $(\diamond)$, and $z_{0}=\langle z\rangle_{0}+6$ bohr $(\mathbf{O})$. Calculations are carried out for perpendicular incidence at $10 \mathrm{meV}$.

behavior could be considered as a precursor for a defected mediated resonance.

In the inset of Fig. 2, several maxima and minima are also found between $E_{z}=0 \mathrm{meV}$ and $E_{z}=10 \mathrm{meV}$. Maxima correspond to nondeflected trajectories $\left(E_{z}=10 \mathrm{meV}\right)$, and minima are due to classical surface rainbows. From both minima, at each side of the adsorbate, the positive and negative values of the rainbow angle, $\theta_{r}$, or critical deflection, can be obtained by means of the relation: $E_{z}^{r}=E_{i} \sin ^{2} \theta_{r}$. However, these rainbows are better characterized from the so-called classical deflection function, $\Delta K$ versus $b$, which provides not only a complementary information about the dynamics of the system but it is the only way to clearly and unambigously assign them. In Fig. 4(a), this function is represented for the case of $E_{i}=10 \mathrm{meV}$. Extrema of such a function give the rainbow angles and the corresponding classical intensities display a singularity (caustics). At negative impact parameters (scattering from the left of the adsorbate), the maximum appears in the single scattering regime (continuous line) and occurs at low $\Delta K$ values; on the contrary, the minimum takes place at the threshold of multiple scattering regime (discontinuous line). The reverse situation is found at positive impact parameters. From this classical analysis, we can affirm that these critical scatterings are, therefore, responsible for the $A_{3}$ peak (low $\Delta K$ values) and the left-hand side shoulder of the $C+D$ peak (high $\Delta K$ values) in Fig. 1(b), althought the corresponding momentum 

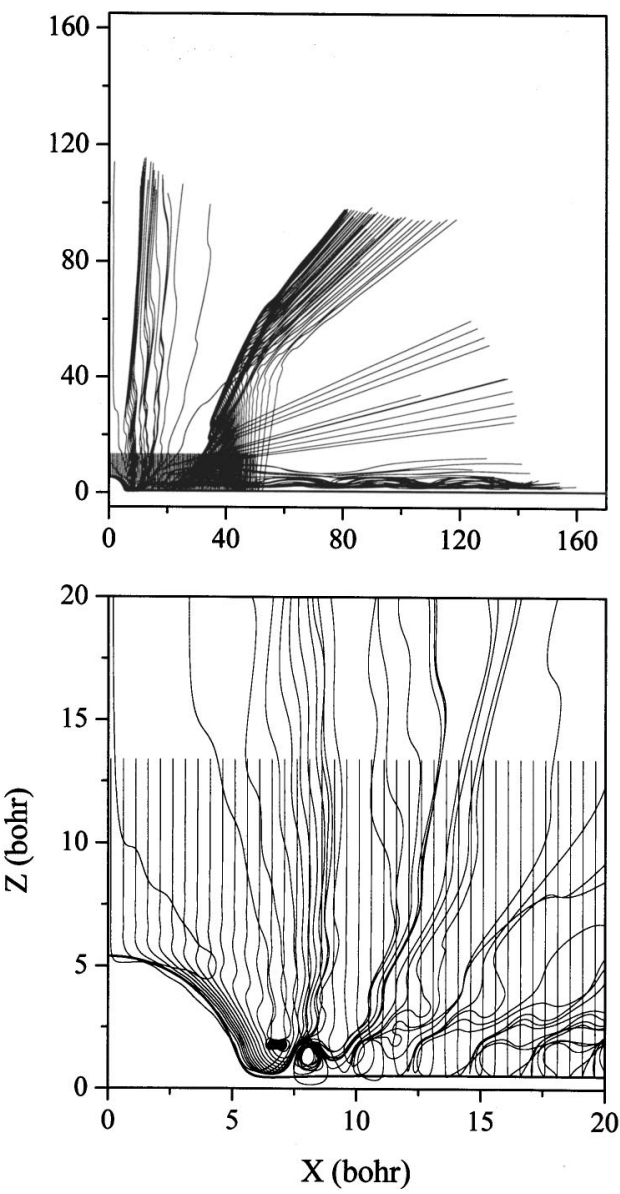

FIG. 5. Quantum trajectories with impact parameters along the slice $z_{0}$ $=\langle z\rangle_{0}-6$ bohr displayed only in the positive region of the $x$ axis, for perpendicular incidence at $10 \mathrm{meV}$. The lower panel shows a magnification close to the adsorbate.

transfer values are very close but not exactly the same as the classical values. As the effective radius of the adsorbate at that incident energy is $5.4 \mathrm{bohr}$, the surface rainbow at low values of $\Delta K$ comes from the clean Pt surface scattering and the second one from the adsorbate scattering before hitting the Pt surface. Notice that no critical feature appears around the $\Delta K$ value for which the $A_{1}$ intensity peak is observed. This fact definitely suppresses the existence of an additional classical surface rainbow. A similar analysis could be done for the scattering at $40 \mathrm{meV}$. For comparison, in Fig. 4(b), the corresponding quantum deflection function ${ }^{31}$ is also plotted. Obviously, all of the impact parameters coming from the different slices of the initial wave packet must contribute to a given value of $\Delta K$ with nonzero intensity in the whole diffraction pattern [see Figs. 1(b)-1( $\left.b^{\prime}\right)$ ]. For this scattering, a quasicontinuous structure is therefore expected. However, in order to see how this deflection function is built, only three ensembles of quantum trajectories have been chosen: Those starting at $z_{0}=\langle z\rangle_{0}-6$ bohr $(\bigcirc), z_{0}=\langle z\rangle_{0}$ bohr $(\diamond)$, and $z_{0}=\langle z\rangle_{0}+6$ bohr $(\bullet)$. As can be clearly seen, the shape of the classical and quantum deflection functions is drastically different. The classical deflection function is continuous except in trapping regions and it displays maximum/minimum structures, whereas the quantum one shows a sort of quanti-
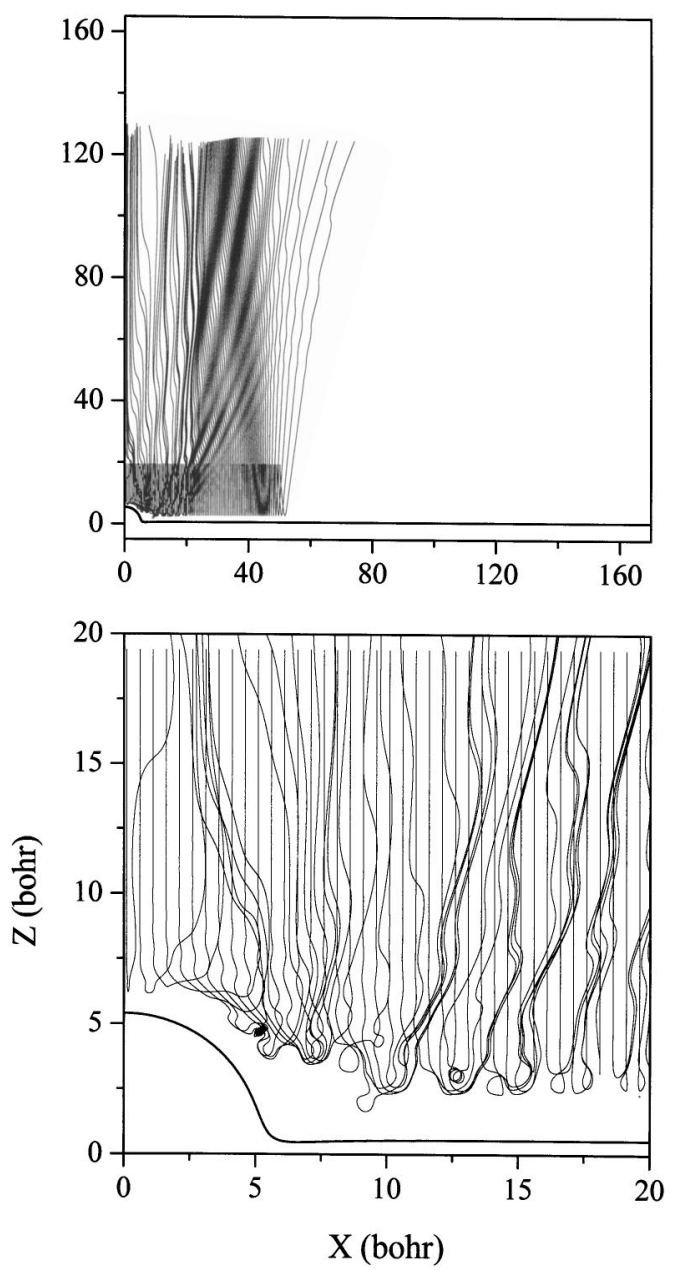

FIG. 6. Quantum trajectories with impact parameters along the slice $z_{0}$ $=\langle z\rangle_{0}$ bohr displayed only in the positive region of the $x$ axis, for perpendicular incidence at $10 \mathrm{meV}$. The lower panel shows a magnification close to the adsorbate.

zation, at values of $\Delta K$ where intensity peaks are observed. Bohmian trajectories belonging to upper slices of the initial plane wave contribute to small values of $\Delta K$, while those corresponding to lower slices contribute to high values of $\Delta K$; in particular, to those values for which surface trapping occurs. The quantum scattering in the classically forbidden region beyond the highest rainbow angle is strongly attenuated due to the fact that a fewer number of quantum trajectories contribute to such intensities. This multilayer-type structure is in sharp contrast to the ladder structure found in the scattering of He atoms from periodic surfaces. ${ }^{31}$ Even more, far from the region influenced by the adsorbate ( $b$ $\geq 15$ bohr), only the specular $\left(\Delta K=0 \mathrm{bohr}^{-1}\right)$ deflection is clearly observed in the classical deflection function. On the contrary, in the quantum domain, nonzero values of the momentum transfer still remain, indicating the nonlocal nature of Bohmian trajectories. The different symmetry found in both functions is also an interesting aspect that we would like to mention. Whereas the quantum deflection function is symmetric with respect to the $b=0$ line, the classical one is symmetric with respect to the origin (inversion symmetry). Finally, it should be noticed that within Bohmian mechanics, 

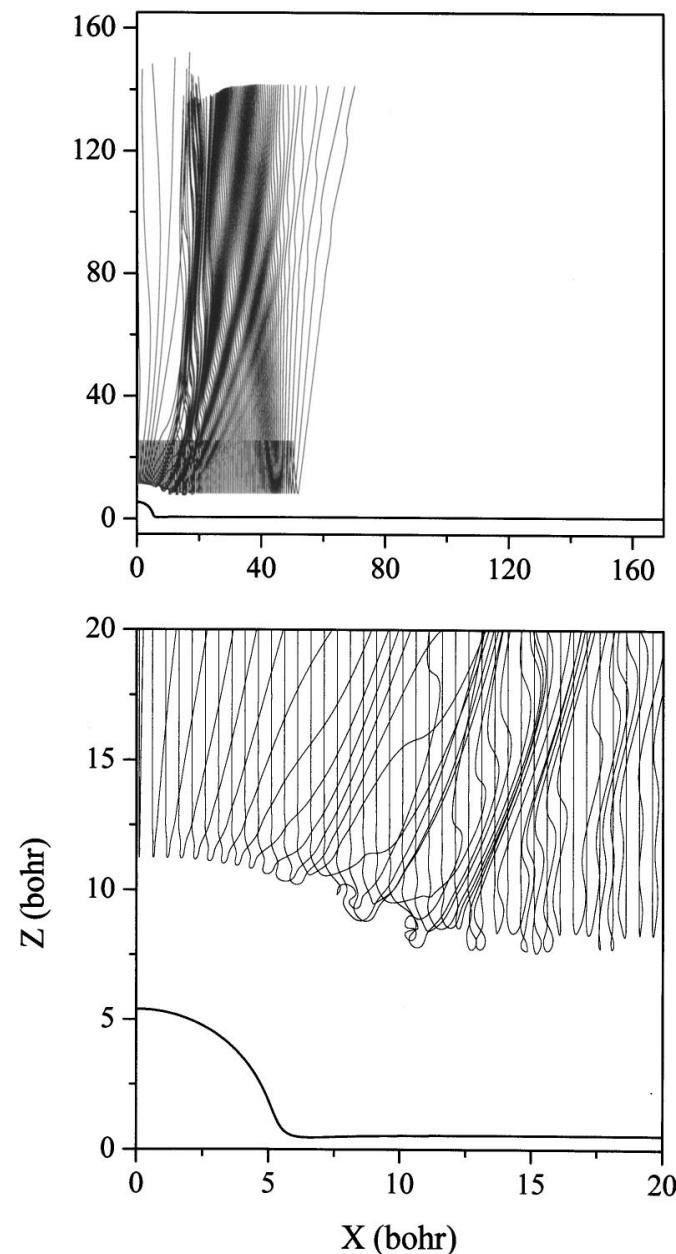

FIG. 7. Quantum trajectories with impact parameters along the slice $z_{0}$ $=\langle z\rangle_{0}+6$ bohr displayed only in the positive region of the $x$ axis, for perpendicular incidence at $10 \mathrm{meV}$. The lower panel shows a magnification close to the adsorbate.

the region of impact parameters where trapping occurs is much larger than the classical one. ${ }^{30}$

One question that still remains open is the interpretation of the $A_{3}$ peak (which can be again seen as a shoulder of the $B$ peak) in the Bohmian mechanics. In order to simplify the presentation of the quantum deflection function, only contributions of quantum trajectories coming from three different slices of the initial wave packet have been plotted which do not show $\Delta K$ values around the $A_{3}$ peak. However, we can provide a pictorial argument to our quantum rainbow assignment. In Figs. 1(c) and 1( $\left.\mathrm{c}^{\prime}\right)$, it is observed that quantum trajectories accumulate in the $B$ direction displaying several foldings due to maximum deflections, a reminiscence of a caustic. Thus, the left-hand side shoulder of the $B$ peak, that is, the $A_{3}$ peak, has to come from the surface rainbow found at low values of $\Delta K$ in Fig. 4(a).

\section{The behavior of quantum trajectories}

An additional feature of quantum trajectories, not mentioned when showing Figs. 1(c) and 1( $\left.\mathrm{c}^{\prime}\right)$, is that they do not behave as straight rays in the interaction region. We have observed that such trajectories follow the boss profile of the adsorbate like in a sliding motion, or spin in loops around
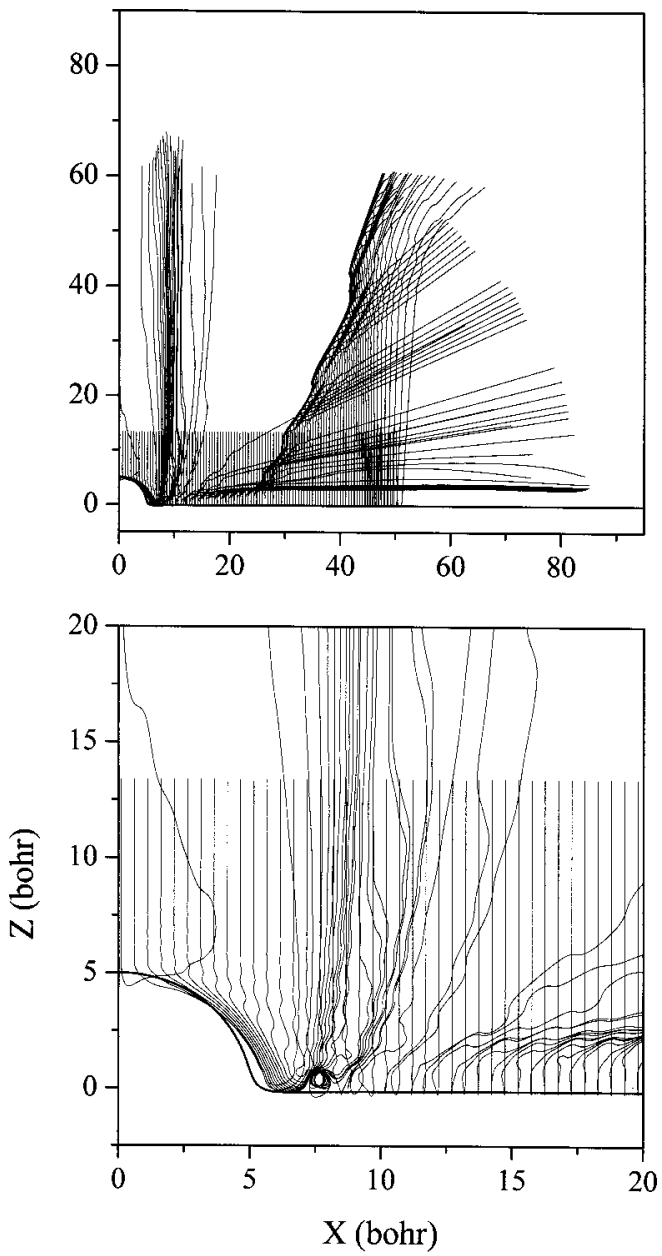

FIG. 8. Quantum trajectories with impact parameters along the slice $z_{0}$ $=\langle z\rangle_{0}-6$ bohr displayed only in the positive region of the $x$ axis, for perpendicular incidence at $40 \mathrm{meV}$. The lower panel shows a magnification close to the adsorbate.

quantum vortices ${ }^{42}$ for some time. Within a hydrodynamical framework, this relates to the existence of a turbulent region surrounding the adsorbate. This is in a sharp contrast with the semiclassical picture used to explain such angular distributions in which rays do not show any of these remarkable features.

In Figs. 5-7 quantum trajectories are displayed for the same three sets of initial conditions considered in Fig. 4(b). Lower panels in Figs. 5-7 are a magnification of the interaction region to clearly see the presence of quantum vortices in this dynamics. Several points are worth commenting here: First, as indicated above, the behavior of the trajectories presented in these figures shows that the quantum dynamics of the system becomes more complex as impact parameters are taken closer to the adsorbate. In a quantum hydrodynamical context, it could be said that trajectories starting from upper slices of the initial wave packet follow a laminar regime, while those starting at lower slices belong to a turbulent one, with a smooth transition between them. Second, this complex dynamics is not chaotic, ${ }^{43}$ but gets highly organized around a number of points avoided by the trajectories (see Fig. 5) and identified as quantum vortices. These quantum vortices are seen to appear evenly spaced at intervals given 

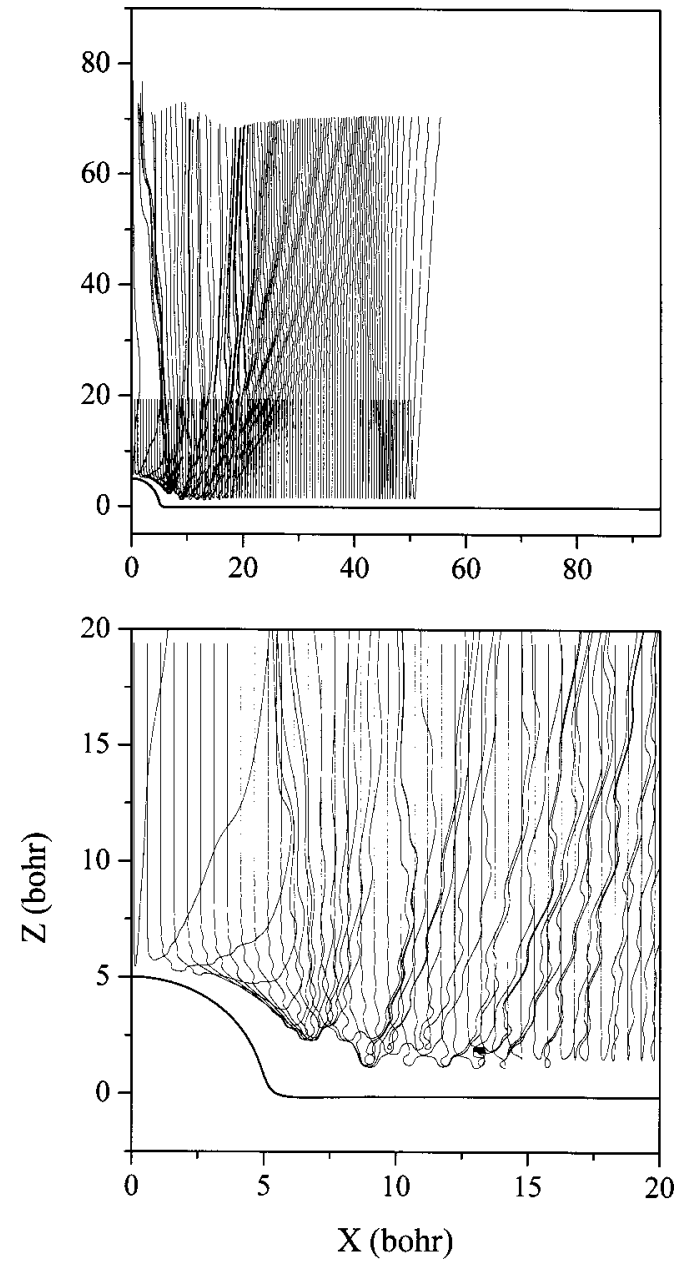

FIG. 9. Quantum trajectories with impact parameters along the slice $z_{0}$ $=\langle z\rangle_{0}$ bohr displayed only in the positive region of the $x$ axis, for perpendicular incidence at $40 \mathrm{meV}$. The lower panel shows a magnification close to the adsorbate.

approximately by $\lambda_{\mathrm{dB}}=2 \pi / k_{i},{ }^{42}$ and are located along straight lines parallel to the clean Pt surface. This vortitial structure leads to a transient trapping regime which should not be confused with the surface trapping associated with the ensemble of trajectories giving rise to the $D$ portion of the final wave packet in Fig. 1(c). In the former case, trapping remains only as the interaction takes place and is due to quantum interference; in the latter, trapping is permanent (unless some adsorbate is present and trajectories will desorb from the surface) and comes from a $z$-to- $x$ momentum exchange. Third, not all quantum trajectories sample the same interaction region but it depends on the departure slice. This is due to the existence of an effective barrier or, alternatively, a quantum pressure (see below) caused by trajectories reaching a deeper distance. ${ }^{35}$ This fact implies a weaker effective interaction acting on the incoming particles initialized in upper slices, their dynamics being much simpler (see Figs. 6 and 7). Hence, the regime is not so turbulent and a wide region of laminar flow is appreciated, although the influence of the vortitial dynamics is still felt. These trajectories contribute mainly to the diffraction peaks labeled as $A$. Fourth, it should be noticed that topologycally quantum trajectories distribute in such a way that they cannot cross through the
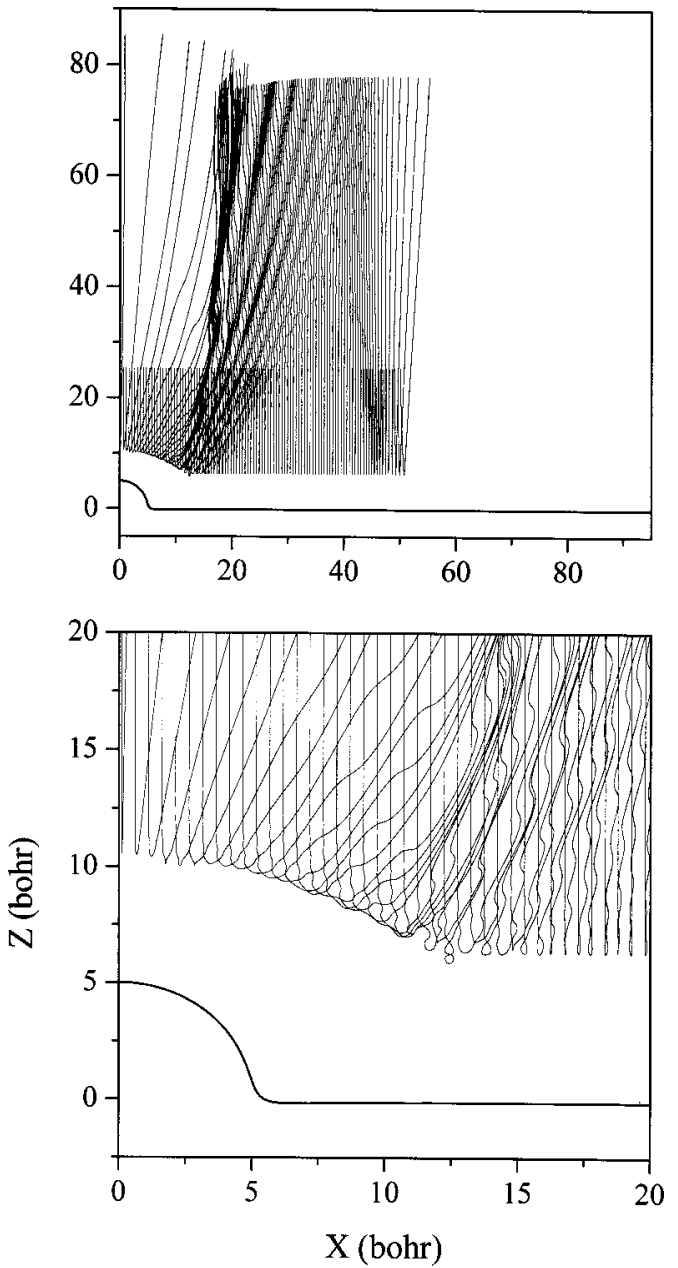

FIG. 10. Quantum trajectories with impact parameters along the slice $z_{0}$ $=\langle z\rangle_{0}+6$ bohr displayed only in the positive region of the $x$ axis, for perpendicular incidence at $40 \mathrm{meV}$. The lower panel shows a magnification close to the adsorbate.

same point at the same time. At each time, the particle momentum in Bohmian mechanics is single valued, ${ }^{18}$ unlike what can be observed in classical mechanics. Thus, due to these avoided crossings, certain ensembles of trajectories can undergo accumulations and foldings evoking caustics (in a classical sense). This fact is particularly interesting at this level because, classically, the inflection points on the potential at each side of the adsorbate cause crossings of the rainbow trajectories in the same position at the same time, unlike quantum trajectories. ${ }^{31}$ As explained in Ref. 30, such a topology can be understood from a detailed analysis of the quantum potential (or, equivalently, the structure of the wave function) and its space-time variation. Therefore, by means of the information transmitted through the quantum potential, a nonlocal causal connection between the region of the (external) potential close to the boss and the rainbow-exit region (as advocated in the classical picture ${ }^{13}$ is established. Fifth, to enhance the capital importance of vorticity, it is possible to know the degree of influence of the transient vortitial dynamics in each peak observed in the intensity pattern. Notice here that the standard quantum mechanics can only give the correct intensity and that, only by means of some semiclassical picture (which is additional, but not self- 

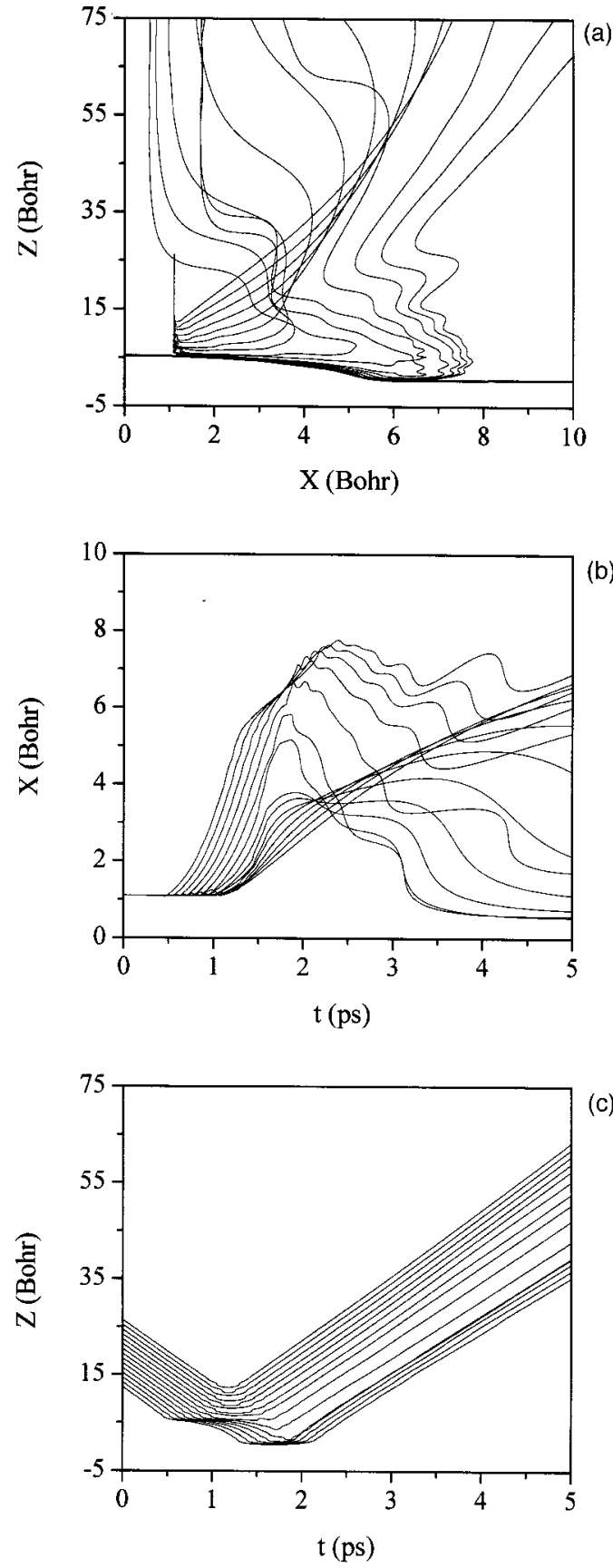

FIG. 11. (a) Quantum trajectories with impact parameters along the slice $x_{0}=1.1 \mathrm{bohr}$ displayed only in the positive region of the $x$ axis, for perpendicular incidence at $10 \mathrm{meV}$. The initial position along $z$ are taken as 12.0 $\leqslant z_{0} \leqslant 22.0$ bohr. The two lower panels show the parallel (b) and perpendicular (c) components of these quantum trajectories as a function of time.

contained in the own structure of quantum mechanics), the dynamical origin of the corresponding peaks is justified. However, the formalism based on quantum trajectories of Bohmian mechanics fulfills both requirements: It reproduces the statistical results provided by the standard theory and, at the same time, it accounts for the dynamical origin of those results without resorting to any argument taken from alternative theoretical formulations. Thus, by binning and weighting the quantum trajectories with an appropriate factor (as described above), the scattering intensity is correctly obtained, as demonstrated in Ref. 30. Proceeding in this way, we have
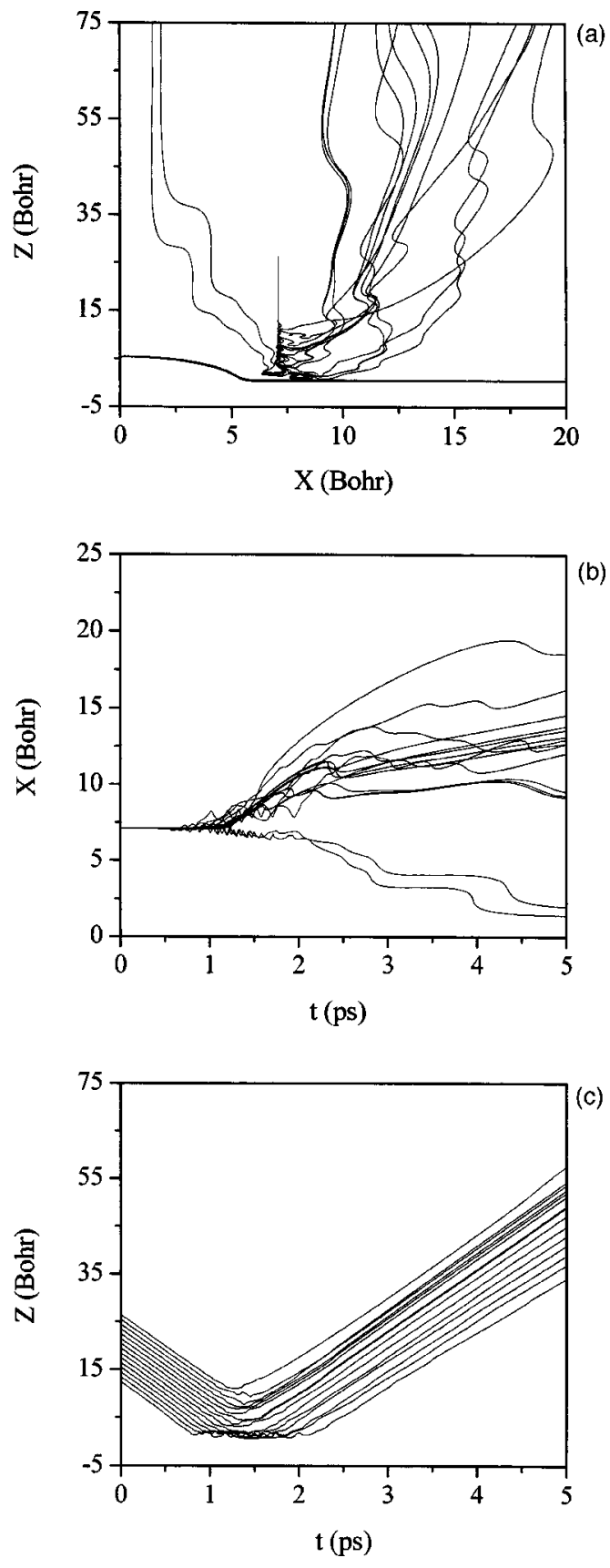

FIG. 12. (a) Quantum trajectories with impact parameters along the slice $x_{0}=7.1$ bohr displayed only in the positive region of the $x$ axis, for perpendicular incidence at $10 \mathrm{meV}$. The initial position along $z$ are taken as 12.0 $\leqslant z_{0} \leqslant 22.0$ bohr. The two lower panels show the parallel (b) and perpendicular (c) components of these quantum trajectories as a function of time.

observed that, at $10 \mathrm{meV}$ and normal incidence, vortitial dynamics contribute to each diffraction peak with the following percentages: $29.9 \%$ for peaks $A, 20.9 \%$ for $B$, and $100 \%$ for both $C$ and $D$. These results show clearly the important role played by the existence of a transient vortitial regime in this type of scattering without the need to evoke any semiclassical picture and so avoiding the controversy found in literature about the interpretation and assignment of the peaks $\left(A_{1}\right.$ and $A_{3}$ ) in the diffraction pattern obtained with this interaction model.

Similar arguments can be used to describe Figs. 8-10, 

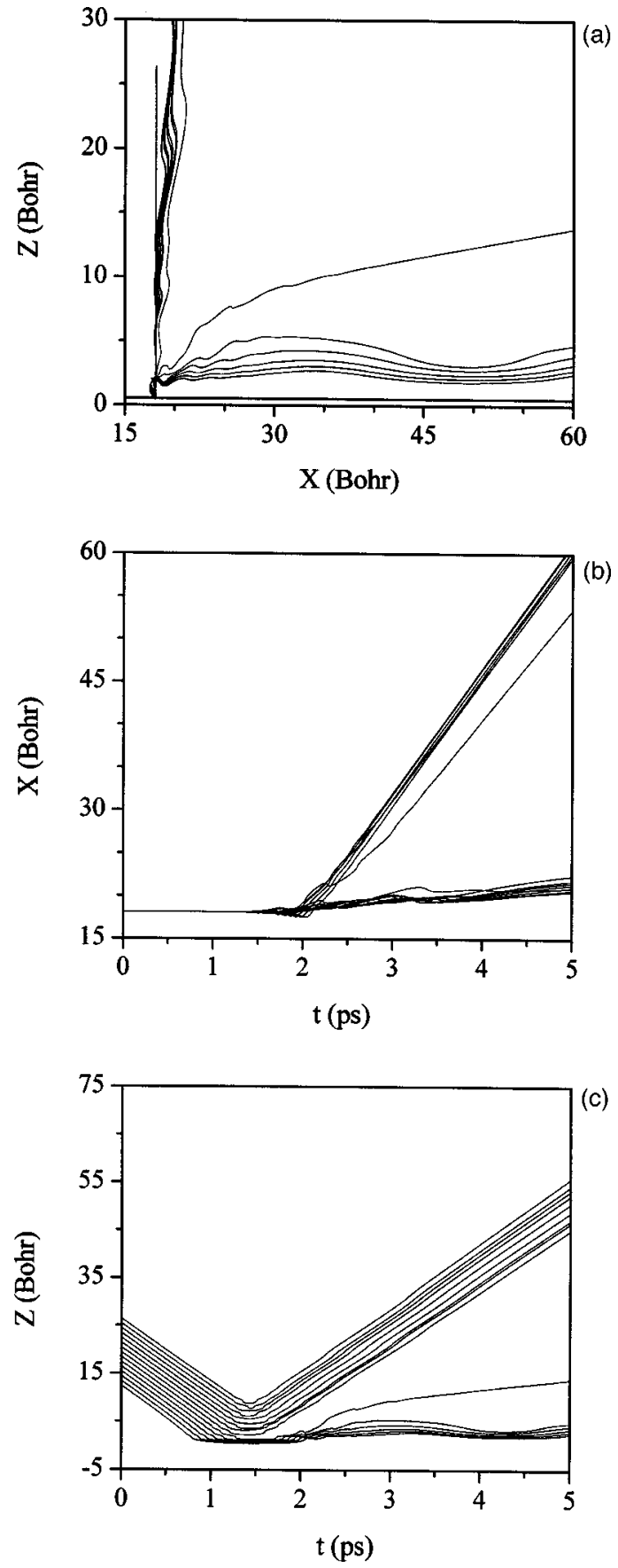

FIG. 13. (a) Quantum trajectories with impact parameters along the slice $x_{0}=18.1$ bohr displayed only in the positive region of the $x$ axis, for perpendicular incidence at $10 \mathrm{meV}$. The initial position along $z$ are taken as $12.0 \leqslant z_{0} \leqslant 22.0$ bohr. The two lower panels show the parallel (b) and perpendicular (c) components of these quantum trajectories as a function of time.

which show the quantum trajectories corresponding to the same slices of the initial wave function along the $x$ direction, but for an incidence of $40 \mathrm{meV}$. However, the most important point here is the observation of a decrease in the influence of the vortitial regime in the dynamics of the system. Notice that, although vortices still appear, the number of trajectories affected by transient trapping is smaller.

To better illustrate the concept of "quantum pressure" introduced above, in Figs. 11-13, quantum trajectories for
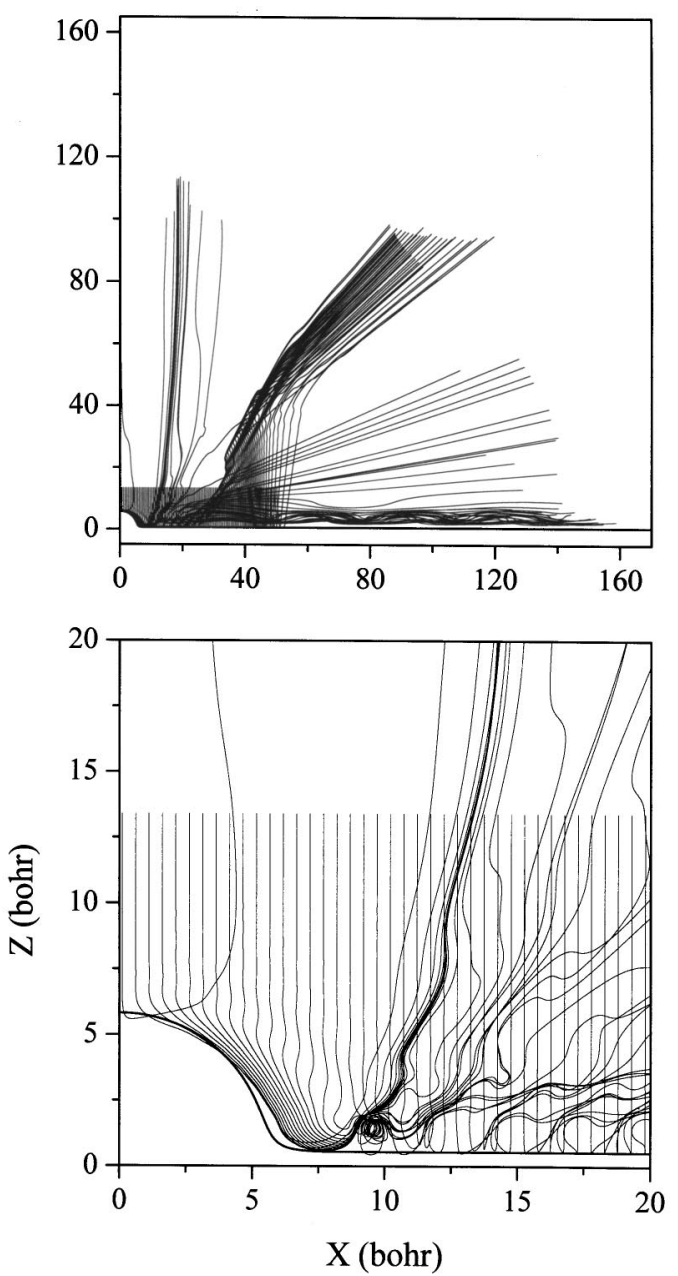

FIG. 14. Quantum trajectories with impact parameters along the slice $z_{0}$ $=\langle z\rangle_{0}-6$ bohr displayed only in the positive region of the $x$ axis, for perpendicular incidence at $10 \mathrm{meV}$, and under the influence of a purely repulsive adsorbate. The lower panel shows a magnification close to the adsorbate.

three different transversal slices of the initial wave function are presented (keeping the same scattering conditions, i.e., perpendicular incidence at $10 \mathrm{meV}$ ). Thus, the initial condition that characterizes each slice is now $x_{0}=1.1,7.1$, and 18.1 bohr, respectively. In Figs. 11-13, panels (b) and (c) show the parallel and perpendicular components of quantum trajectories as a function of time, respectively. From an inspection of panel (c), those quantum trajectories initiated closer to the adsorbate or surface "create" an artificial barrier that prevents other trajectories initiated further away to reach the surface. Let us remark the close resemblance between the deflection of these trajectories during the interaction, and that shown by the trajectories corresponding to the scattering of a Gaussian wave packet off a hard wall. ${ }^{44}$ Furthermore, among those trajectories belonging to the slice $x_{0}$ $=1.1 \mathrm{bohr}$, it is possible to distinguish double and single collisions [see panel (c)]. In the former case, atoms first hit the adsorbate and then they slide along its surface to collide with the Pt surface, before escaping from the interaction region. In the latter case, trajectories display only a single collision (with the adsorbate) avoiding inner trajectories close to the adsorbate surface. Moreover, surface trapping can be 

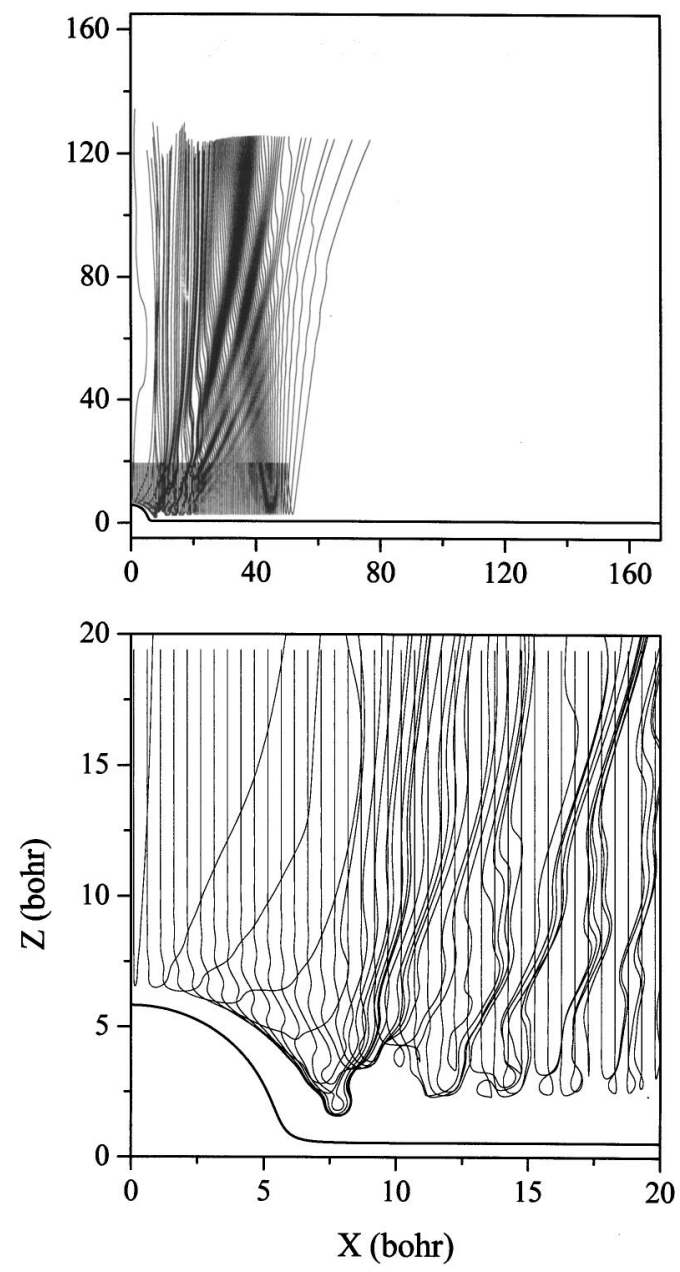

FIG. 15. Quantum trajectories with impact parameters along the slice $z_{0}$ $=\langle z\rangle_{0}$ bohr displayed only in the positive region of the $x$ axis, for perpendicular incidence at $10 \mathrm{meV}$, and under the influence of a purely repulsive adsorbate. The lower panel shows a magnification close to the adsorbate.

again seen in Fig. 13 [see panel (b)] where the parallel components of the trajectories follow a uniform motion (constant velocity). Notice also that, as in the case of free motion along the $z$ direction [see Figs. 11(c) and 12(c)], the condition given by Eq. (10) is fulfilled, and a quantum asymptotic motion is also observed along the $x$-direction. ${ }^{41}$

Finally, in order to complete the Bohmian description of our system, in Figs. 14 and 15 the effect of a purely repulsive $\mathrm{He}-\mathrm{CO}$ potential on the scattering at perpendicular incidence and energy of $10 \mathrm{meV}$ is shown. The quantum trajectories plotted are initiated at the slices $z_{0}=\langle z\rangle_{0}-6 \mathrm{bohr}$ and $z_{0}$ $=\langle z\rangle_{0}$, respectively. Surprisingly, a vortitial regime still remains close to the adsorbate although, by comparison with their counterparts (see Figs. 5 and 6), the turbulent region is more diluted. In particular, the accumulation and foldings of the quantum trajectories are also diminished. This fact leads to the suppression of the corresponding contributions to the $A_{3}$ peak in the diffraction pattern. The absence of the $A_{1}$ peak and the left-hand side shoulder of the $C+D$ peak can also be explained with similar arguments. For example, notice that the number of nearly nondeflected trajectories in Fig. 14 is much less than in Fig. 5.

\section{CONCLUSIONS}

To summarize, in this work, we have presented a complete theoretical study of atomic scattering from single adsorbates on a metallic surface within the Bohmian formalism, which provides an intuitive, causal description of this diffraction in terms of quantum trajectories. The interaction model chosen for this scattering presents classical rainbow features (extrema in the classical deflection function) and surface trapping. In this formalism, rainbow features are associated to the whole diffraction pattern rather than to individual peaks ${ }^{31}$ unlike the proposal based on classical mechanisms. ${ }^{13}$ This is due to the nonlocal and contextdependent character of the quantum potential, which makes the adsorbate extend its influence further away than its classical range of interaction.

On the other hand, the quantum process takes place through rich dynamics, in which the concept of transient vortitial trapping plays a key role. In this sense, we have been able to provide an extended and more complete description of the corresponding scattering process than that existing in literature in terms of a classical or semiclassical picture. The use of quantum trajectories to better interpret and assign the dynamical origin of the intensity peaks displayed in the diffraction pattern has been determinant. In particular, and within this interaction model, the controversial interpretation appearing in literature about the assignment of the intensity peaks (mainly, the $A_{1}$ and $A_{3}$ peaks) dilutes. The reflection symmetry interference notion, originally introduced to explain certain oscillations in the experimental diffraction patterns, is not necessary within the Bohmian formalism.

\section{ACKNOWLEDGMENTS}

This work has been supported by MCyT (Spain) under Contract Nos. BFM2000-347 and BFM2001-2179. One of the authors (A.S.S.) gratefully acknowledges a doctoral grant from the Consejería de Educación y Cultura of the Comunidad Autónoma de Madrid (Spain).

${ }^{1}$ B. Poelsema and G. Comsa, Scattering of Thermal Energy Atoms from Disordered Surfaces, Springer Tracts in Modern Physics, Vol. 115 (Springer, Berlin, 1989).

${ }^{2}$ E. Hulpke, Helium Atom Scattering from Surfaces (Springer, Berlin, 1992).

${ }^{3}$ D. Farías and K. H. Rieder, Rep. Prog. Phys. 61, 1575 (1998), and references therein.

${ }^{4}$ A. S. Sanz, F. Borondo, and S. Miret-Artes, in J. Phys.: Condens. Matter, special issue on "Surface Dynamics: Phonons, Adsorbate Vibrations and Diffusion," edited by D. Farias, E. Garcia-Michel, and S. Miret-Artes (2002).

${ }^{5}$ J. P. Toennies, in Surface Phonons, Springer Series in Surface Sciences Vol. 21, edited by W. Kress and F. W. de Wette (Springer, Berlin, 1991), p. 111.

${ }^{6}$ B. Gumhalter, Phys. Rep. 351, 1 (2001).

${ }^{7}$ J. A. Stinnett, R. J. Madix, and J. C. Tully, J. Chem. Phys. 104, 3134 (1996).

${ }^{8}$ F. Borondo, R. Guantes, J. Bowers, C. Jaffé, and S. Miret-Artés, in Hamiltonian Systems with Three and More Degrees of Freedom, edited by C. Simó (Kluwer, Netherlands, 1999), p. 314.

${ }^{9}$ S. Miret-Artés, Surf. Sci. 339, 205 (1995); 366, L681 (1996); 366, L735 (1996).

${ }^{10}$ A. M. Lahee, J. R. Manson, J. P. Toennies, and C. Wöll, Phys. Rev. Lett. 57, 471 (1986); J. Chem. Phys. 86, 7194 (1987).

${ }^{11}$ G. Drolshagen and R. Vollmer, J. Chem. Phys. 87, 4948 (1987). 
${ }^{12}$ A. P. Graham, F. Hofman, J. P. Toennies, and J. R. Manson, J. Chem. Phys. 105, 2093 (1996).

${ }^{13}$ A. T. Yinnon, R. Kosloff, and R. B. Gerber, J. Chem. Phys. 88, 7209 (1988).

${ }^{14}$ M. Carré and D. Lemoine, J. Chem. Phys. 101, 5305 (1994).

${ }^{15}$ D. Lemoine, Phys. Rev. Lett. 81, 461 (1998).

${ }^{16}$ B. H. Choi, A. P. Graham, K. T. Tang, and J. P. Toennies, J. Chem. Phys. 112, 10538 (2000).

${ }^{17}$ D. Bohm, Phys. Rev. 85, 166 (1952).

${ }^{18} \mathrm{P}$. R. Holland, The Quantum Theory of Motion (Cambridge University Press, Cambridge, UK, 1993).

${ }^{19}$ E. Madelung, Z. Phys. 40, 332 (1926).

${ }^{20}$ L. de Broglie, Compt. Rend. 183, 447 (1926); 184, 273 (1927).

${ }^{21}$ Y. Aharonov and D. Bohm, Phys. Rev. 115, 485 (1955).

${ }^{22}$ F. London, Rev. Mod. Phys. 17, 310 (1945).

${ }^{23}$ A. L. Fetter and A. A. Svidzinsky, J. Phys. C 13, R135 (2001).

${ }^{24}$ P. A. M. Dirac, Proc. R. Soc. London, Ser. A 133, 60 (1931).

${ }^{25}$ I. Bialynicki-Birula and Z. Bialynicka-Birula, Phys. Rev. D 3, 2410 (1971).

${ }^{26}$ J. Riess, Phys. Rev. D 2, 647 (1970).

${ }^{27}$ J. O. Hirschfelder, C. J. Goebel, and L. W. Bruch, J. Chem. Phys. 61, 5456 (1974).

${ }^{28}$ H. Wu and D. W. L. Sprung, Phys. Rev. A 49, 4305 (1994).

${ }^{29}$ C. L. Lopreore and R. E. Wyatt, Phys. Rev. Lett. 82, 5190 (1999); Chem. Phys. Lett. 325, 73 (2000).

${ }^{30}$ A. S. Sanz, F. Borondo, and S. Miret-Artés, Phys. Rev. B 61, 7743 (2000).
${ }^{31}$ A. S. Sanz, F. Borondo, and S. Miret-Artés, Europhys. Lett. 55, 303 (2001).

${ }^{32}$ O. V. Prezhdo and C. Brooksby, Phys. Rev. Lett. 86, 3215 (2001).

${ }^{33}$ E. Gindensperger, C. Meier, and J. A. Beswick, J. Chem. Phys. 113, 9369 (2000); 116, 8 (2002).

${ }^{34}$ E. Gindensperger, C. Meier, J. A. Beswick, and M.-C. Heitz, J. Chem. Phys. 116, 10051 (2002).

${ }^{35}$ A. S. Sanz, F. Borondo, and S. Miret-Artés, J. Phys. C 14, 6109 (2002).

${ }^{36}$ V. Allori, D. Dürr, S. Goldstein, and N. Zanghí, J. Opt. B: Quantum Semiclassical Opt. 4, 482 (2002).

${ }^{37}$ D. Kosloff and R. Kosloff, J. Comput. Phys. 52, 35 (1983).

${ }^{38}$ H. Tal-Ezer and R. Kosloff, J. Chem. Phys. 81, 3967 (1984)

${ }^{39}$ R. Kosloff, Dynamics of Molecules and Chemical Reactions, edited by R. E. Wyatt and J. Z. Zhang (Marcel Dekker, New York, 1996), p. 185.

${ }^{40}$ A. Glebov, J. R. Manson, J. Skofronick, and J. P. Toennies, Phys. Rev. Lett. 78, 1508 (1997).

${ }^{41}$ The concept of asymptotic components of the energy in this case refers to the situation in which the motion can be decoupled along each direction, but does not necessarily imply that atoms move freely. Indeed, they move freely only along the parallel direction when surface trapping occurs.

${ }^{42}$ A. S. Sanz, F. Borondo, and S. Miret-Artés, Phys. Rev. B 69, 115413 (2004).

${ }^{43}$ R. Guantes, A. S. Sanz, J. Margalef-Roig, and S. Miret-Artés, Surf. Sci. Rep. (to be published).

${ }^{44}$ C. Dewdbey and B. J. Hiley, Found. Phys. 12, 27 (1982). 